\title{
ON THE OSCILLATION OF DIFFERENTIAL EQUATIONS WITH AN OSCILLATORY COEFFICIENT
}

\author{
B. J. HARRIS AND Q. KONG
}

\begin{abstract}
We derive lower bounds for the distance between consecutive zeros of solutions of

$$
y^{\prime \prime}+q(t) y=0
$$

when $q$ takes both positive and negative values. We apply our results to the limit point/limit circle classifications of $\left(^{*}\right)$.
\end{abstract}

\section{INTRODUCTION}

We consider the linear, second order differential equation

$$
y^{\prime \prime}+q(t) y=0
$$

where we suppose that $q$ is a real-valued member of $L_{\text {loc }}^{1}$.

The question that we consider here is that of determining a lower bound for the distance between consecutive zeros of a solution of (1.1). Perhaps the best known existing result of this type is due to Lyapunov.

Theorem A. Let $q$ be continuous on $[a, b]$. A necessary condition for a nontrivial solution of (1.1) to have two zeros in $[a, b]$ is that

$$
\int_{a}^{b} q^{+}(t) d t>\frac{4}{b-a} \text { where } q^{+}(t):=\max (0, q(t)) .
$$

A proof of this result may be found in the books [1] and [3] and extensions are given in [2] and [4].

Intuitively, one would expect that if $q$ had a large negative part, or if $q$ were oscillatory, then the distance between consecutive zeros would tend to become larger. Theorem A would be unable to detect this difference. Our object in this paper is to extend Theorem A in such a way as to use the negative part of $q$ to obtain a keener bound. This is achieved in Corollary 2.2 below.

We also demonstrate, by means of Examples 2.1 and 3.1, the rather surprising fact that, under certain circumstances, the greater the frequency of oscillation of $q$, the smaller the frequency of oscillation of solutions of (1.1). This effect could not have been observed by previous results.

Received by the editors March 16,1994; originally communicated to the Proceedings of the AMS by Hal S. Smith.

1991 Mathematics Subject Classification. Primary 34C10. 
Lower bounds for the distance between successive zeros of non-trivial solutions of (1.1) have application to the limit point/limit circle classification of (1.1). This connection was established by Patula and Wong in [6] and exploited by Yan in [7].

In $\S 3$ we use our results to derive simple criteria for (1.1) to be the limit point at infinity.

\section{Preliminary Results}

Lemma 2.1. If $y$ is a solution of (1.1) satisfying $y^{\prime}(d)=0, y(b)=0$, and $y(t)>0$ and $y^{\prime}(t) \leq 0$ for $t \in(d, b)$, then

$$
\sup _{d \leq t \leq b} \int_{d}^{t} q(s) d s>0 .
$$

Proof. Suppose the contrary. Then $\int_{d}^{t} q(s) d s \leq 0$ for $t \in[d, b]$. We let $Q(t):=\int_{d}^{t} q(s) d s$, and we define the Riccati variable

$$
r(t):=-y^{\prime}(t) / y(t)
$$

We thus have $r(d)=0, \lim _{t \rightarrow b^{-}} r(t)=\infty$ and $r(t) \geq 0$ for $t \in(d, b)$. It follows from (1.1) that

$$
r^{\prime}(t)=q(t)+r(t)^{2}
$$

whence

$$
r(t)=Q(t)+\int_{d}^{t} r(s)^{2} d s .
$$

In a similar way, if $z$ is the non-trivial solution of the equation $z^{\prime \prime}=0$ with $z^{\prime}(d)=0$ and $R(t):=-z^{\prime}(t) / z(t)$, then

$$
R(t)=\int_{d}^{t} R(s)^{2} d s
$$

so that $R(t)=0$ for all $t \in[d, \infty)$. As a simple consequence of the general theory of integral inequalities (see [4, Lemma 3]) we see that $r(t) \leq R(t)=0$ for $t \in[d, b)$, thus contradicting the fact that $\lim _{t \rightarrow b^{-}} r(t)=\infty$. The proof is now complete.

Theorem 2.1. Let $y$ denote a non-trivial solution of (1.1) satisfying $y^{\prime}(d)=$ $0, y(b)=0$, and $y(t) \neq 0$ for $t \in[d, b)$. Then

$$
(b-d) \sup _{d \leq t \leq b}\left|\int_{d}^{t} q(s) d s\right|>1 .
$$

Moreover, if there are no extreme values of $y$ in $(d, b)$, then

$$
(b-d) \sup _{d \leq t \leq b} \int_{d}^{t} q(s) d s>1 .
$$


Proof. We assume, without loss of generality, that $y(t)>0$ for $t \in[d, b)$. With $r$ defined by (2.1) we set

$$
w(t):=\int_{d}^{t} r(s)^{2} d s \text { for } t \in[d, b) .
$$

Thus, $r(d)=w(d)=0$ and from (2.2) $\lim _{t \rightarrow b^{-}} r(t)=\lim _{t \rightarrow b^{-}} w(t)=\infty$ because

$$
r(t)=\int_{d}^{t} q(s) d s+w(t) \quad \text { for } t \in[d, b) .
$$

We set $Q^{*}:=\sup _{d \leq t \leq b}\left|\int_{d}^{t} q(s) d s\right|$ and observe that

$$
|r(t)| \leq Q^{*}+w(t)
$$

so that

$$
w^{\prime}(t)=r(t)^{2} \leq\left(Q^{*}+w(t)\right)^{2}
$$

and

$$
\frac{w^{\prime}(t)}{\left(Q^{*}+w(t)\right)^{2}} \leq 1
$$

Integrating $(2.7)$ over $[d, b]$ we obtain

$$
-\left.\frac{1}{Q^{*}+w(t)}\right|_{d} ^{b} \leq b-d,
$$

which implies that $1 / Q^{*} \leq b-d$ or $(b-d) Q^{*} \geq 1$. We remark that equality cannot hold, for otherwise $|Q(t)|=\left|\int_{d}^{t} q(s) d s\right|=Q^{*}$ a.e. on $[d, b)$, which contradicts the fact that $Q$ is continuous and $Q(d)=0$.

If $d$ is the largest extreme point of $y$ in $[d, b)$, then $y^{\prime}(t) \leq 0$ and thus $r(t) \geq 0$ for $t \in[d, b)$. We set $Q_{*}:=\sup _{d \leq t \leq b} \int_{d}^{t} q(s) d s$. By Lemma 2.1, $Q_{*}>0$; and from (2.6)

$$
0 \leq r(t) \leq Q_{*}+w(t) .
$$

The proof of the second part of the theorem now follows in a way similar to that of the first.

Theorem 2.2. Let $y$ denote a non-trivial solution of (1.1) satisfying $y(a)=0$, $y^{\prime}(c)=0$, and $y(t) \neq 0$ for $t \in(a, c]$. Then

$$
(c-a) \sup _{a \leq t \leq c}\left|\int_{t}^{c} q(s) d s\right|>1 .
$$

Moreover, if there are no extreme values of $y$ in $(a, c)$, then

$$
(c-a) \sup _{a \leq t \leq c} \int_{t}^{c} q(s) d s>1 .
$$

The proof of this result is similar to the proof of Theorem 2.1 and is omitted. 
Corollary 2.1. If

$$
(b-d) \sup _{d \leq t \leq b}\left|\int_{d}^{t} q(s) d s\right| \leq 1,
$$

then (1.1) is right disfocal on $[d, b)$; if

$$
(c-a) \sup _{a \leq t \leq c}\left|\int_{t}^{c} q(s) d s\right| \leq 1,
$$

then (1.1) is left disfocal on $(a, c]$. (See $[4, p .486]$ for the definition of (1.1) to be right or left disfocal on an interval.)

Theorem 2.3. Let $a$ and $b$ denote two consecutive zeros of a non-trivial solution, $y$, of (1.1). Then there exist two disjoint subintervals of $[a, b], I_{1}$ and $I_{2}$, satisfying

$$
(b-a) \int_{I_{1} \cup I_{2}} q(s) d s>4
$$

and

$$
\int_{[a, b] \backslash\left(I_{1} \cup I_{2}\right)} q(s) d s \leq 0 .
$$

Proof. Let $c$ and $d$ denote the least and greatest extreme points of $y$ on $[a, b]$, respectively. If there is only one zero of $y^{\prime}$ in $(a, b)$, then $c$ and $d$ coincide. Then $y^{\prime}(d)=0, y(b)=0$, and $y^{\prime}(t) \neq 0$ for $t \in(d, b]$. By Theorem 2.1 inequality (2.4) holds. There thus exists $b_{1} \in(d, b]$ such that

$$
\int_{d}^{b_{1}} q(s) d s>\frac{1}{b-d} \text { and } \int_{d}^{b_{1}} q(s) d s \geq \int_{d}^{b} q(s) d s .
$$

Similarly, we can choose $a_{1} \in[a, c)$ such that

$$
\int_{a_{1}}^{c} q(s) d s>\frac{1}{c-a} \text { and } \int_{a_{1}}^{c} q(s) d s \geq \int_{a}^{c} q(s) d s .
$$

Let $I_{1}:=\left[d, b_{1}\right]$ and $I_{2}:=\left[a_{1}, c\right]$, then

$$
\begin{aligned}
(b-a) \int_{I_{1} \cup I_{2}} q(s) d s & \geq[(b-d)+(c-a)]\left(\int_{d}^{b_{1}} q(s) d s+\int_{a_{1}}^{c} q(s) d s\right) \\
& >[(b-d)+(c-a)]\left(\frac{1}{b-d}+\frac{1}{c-a}\right) \\
& =2+\frac{c-a}{b-d}+\frac{b-d}{c-a} \geq 4
\end{aligned}
$$

and (2.10) is verified. It is also easy to see that $\int_{b_{1}}^{b} q(s) d s \leq 0$ and $\int_{a}^{a_{1}} q(s) d s \leq 0$. To verify (2.11) it is sufficient to show that $\int_{c}^{d} q(s) d s \leq 0$. In 
fact, since $y^{\prime}(c)=y^{\prime}(d)=0$, we have $r(c)=r(d)=0$. From (2.2),

$$
0=r(d)-r(c)=\int_{c}^{d} q(s) d s+\int_{c}^{d} r(s)^{2} d s
$$

This means that $\int_{c}^{d} q(s) d s \leq 0$ and hence that (2.11) holds.

Corollary 2.2. Suppose that for every two disjoint subintervals, $I_{1}$ and $I_{2}$, of $[\alpha, \beta]$, we have

$$
(\beta-\alpha) \int_{I_{1} \cup I_{2}} q(s) d s \leq 4
$$

Then equation (1.1) is disconjugate on $[\alpha, \beta]$.

Proof. Suppose the contrary, then there exists a non-trivial solution $y$ of (1.1) with $y(a)=y(b)=0$ for $\alpha \leq a<b \leq \beta$. Without loss of generality we assume that $y(t) \neq 0$ for $t \in(a, b)$. By Theorem 2.3, there exist two disjoint intervals, $I_{1}$ and $I_{2}$, of $[a, b] \subset[\alpha, \beta]$ with

$$
(b-a) \int_{I_{1} \cup I_{2}} q(s) d s>4 .
$$

Hence, $(\beta-\alpha) \int_{I_{1} \cup I_{2}} q(s) d s>4$, which gives a contradiction.

Corollary 2.3. Suppose that a non-trivial solution of (1.1) has $N$ zeros in $[a, b]$ for $N \geq 2$. There exist $2 N$ disjoint subintervals of $[a, b], I_{i j}$ for $i=$ $1, \ldots, N, j=1,2$, such that

$$
N<\frac{1}{2}\left[(b-a) \int_{I} q(s) d s\right]^{1 / 2}+1
$$

and

$$
\int_{[a, b] \backslash I} q(s) d s \leq 0
$$

where $I:=\bigcup_{i=1}^{N} \bigcup_{j=1}^{2} I_{i j}$.

We remark that Corollary 2.3 provides an extension of [3, Corollary 5.2] in that we use the negative part of $q$ to achieve a sharper bound.

Proof. Let $t_{i}, i=1, \ldots, N$, be the zeros of $y$ in $[a, b]$. By Theorem 2.3, for $i=1, \ldots, N-1$ there are two disjoint subintervals of $\left[t_{i}, t_{i+1}\right], I_{i 1}$ and $I_{i 2}$, with

$$
\int_{I_{i 1} \cup I_{i 2}} q(s) d s>\frac{4}{t_{i+1}-t_{i}}
$$

and

$$
\int_{\left[t_{i}, t_{i+1}\right] \backslash\left(I_{i 1} \cup I_{i 2}\right)} q(s) d s \leq 0
$$


We sum (2.15) for $i$ from 1 to $N-1$ and see that

$$
\int_{I} q(s) d s>4 \sum_{i=1}^{N-1} \frac{1}{t_{i+1}-t_{i}}
$$

and by the inequality for the harmonic mean

$$
\int_{I} q(s) d s>\frac{4(N-1)^{2}}{t_{N}-t_{1}} \geq \frac{4(N-1)^{2}}{b-a}
$$

whence,

$$
(N-1)^{2}<\frac{b-a}{4} \int_{I} q(s) d s .
$$

This implies (2.13). From (2.16) it is easy to deduce (2.14).

In fact, (2.14) is true for the case that $a=t_{1}$ and $b=t_{N}$. For the case that $a<t_{1}$, let $I_{11}=\left[s_{1}, s_{2}\right]$ be the first disjoint subinterval of $I$. If $\int_{a}^{s_{1}} q(s) d s>0$, then replace $I_{11}$ in $I$ by $I_{11}^{\prime}=\left[a, s_{2}\right]$. Similar manipulation can be done for the case that $b>t_{N}$. Then for the new $I$ both (2.13) and (2.14) are satisfied.

Example 2.1. Consider the equation

$$
y^{\prime \prime}+(\cos k t) y=0, \text { for } k>0 \text {. }
$$

Conclusion 1. Equation (2.17) is right disfocal on $[0, b)$ and left disfocal on $(0, b]$ if $0<b \leq \frac{k}{2}$.

Conclusion 2. Equation (2.17) is disconjugate on $[0, b]$ if $0<b \leq k$.

Proof. We set $q(t):=\cos (k t)$ and suppose that $0<b \leq \frac{k}{2}$. Then

$$
\sup _{0 \leq t \leq b}\left|\int_{0}^{t} q(s) d s\right|=\sup _{0 \leq t \leq b}\left|\int_{0}^{t} \cos (k s) d s\right| \leq \frac{2}{k} .
$$

Thus

$$
b \sup _{0 \leq t \leq b}\left|\int_{0}^{t} q(s) d s\right| \leq 1 .
$$

By Corollary 2.1, equation (2.17) is right disfocal. It may be shown in a similar way that it is also left disfocal. Suppose now that $0<b \leq k$. In (2.12)

$$
\int_{I_{1} \cup I_{2}} q(s) d s=\int_{I_{1} \cup I_{2}} \cos (k s) d s \leq \frac{4}{k}
$$

Thus,

$$
b \int_{I_{1} \cup I_{2}} q(s) d s \leq 4 .
$$

By Corollary 2.2, equation (2.17) is disconjugate on $[0, b]$.

\section{THE DISTANCE BETWEEN CONSECUTIVE ZEROS}

For any $t$ and $\delta>0$ we denote by $\left(I_{1} \cup I_{2}\right)(t, \delta)$ the union of two disjoint subintervals of $[t, t+\delta], I_{1}$ and $I_{2}$. 
Theorem 3.1. Let $y$ denote an oscillatory solution of (1.1). If

$$
\limsup _{t \rightarrow \infty}\left(\delta \int_{I_{1} \cup I_{2}(t, \delta)} q(s) d s\right)<4
$$

for all $\delta>0$ and for every two disjoint subintervals, $I_{1}$ and $I_{2}$ of $[t, t+\delta]$, then the distance between consecutive zeros of $y$ is unbounded as $t \rightarrow \infty$.

Proof. We suppose for a contradiction that (1.1) has a solution, $y$, whose sequence of zeros $\left\{t_{n}\right\}_{n=1}^{\infty}$ contains a subsequence $\left\{t_{n_{k}}\right\}_{k=1}^{\infty}$ such that $0<$ $t_{n_{k+1}}-t_{n_{k}} \leq \delta$ for some $\delta$ and all $k$. By Theorem 2.3 there are disjoint subintervals of $\left[t_{n_{k}}, t_{n_{k+1}}\right], I_{1}\left(n_{k}, \delta\right)$ and $I_{2}\left(n_{k}, \delta\right)$, such that

$$
\left(t_{n_{k+1}}-t_{n_{k}}\right) \int_{\left(I_{1} \cup I_{2}\right)\left(n_{k}, \delta\right)} q(s) d s>4 \text { for all } k .
$$

This implies that

$$
\delta \int_{\left(I_{1} \cup I_{2}\right)\left(n_{k}, \delta\right)} q(s) d s>4 \text { for all } k,
$$

which contradicts (3.1) and completes the proof.

Theorem 3.2. Let $y$ denote an oscillatory solution of (1.1). If there exists $\delta_{0}>0$ such that

$$
\lim _{t \rightarrow \infty} \int_{\left(I_{1} \cup I_{2}\right)\left(t, \delta_{0}\right)} q(s) d s=0
$$

for every two disjoint intervals $I_{1}$ and $I_{2}$ of $\left[t, t+\delta_{0}\right]$, then the distance between consecutive zeros of $y$ is unbounded as $t \rightarrow \infty$.

Proof. We first show that for all $\delta>0$ and any disjoint intervals $I_{1}$ and $I_{2}$ of $[t, t+\delta]$

$$
\lim _{t \rightarrow \infty} \int_{\left(I_{1} \cup I_{2}\right)(t, \delta)} q(s) d s=0 .
$$

Let $k$ denote the least integer with $k \delta_{0} \geq \delta$, and $t_{i}=t+i \delta_{0}, i=0,1, \ldots$, $k-1$, and $t_{k}=\delta$; then

$$
\int_{\left(I_{1} \cup I_{2}\right)(t, \delta)} q(s) d s=\sum_{i=0}^{k-1} \int_{\left(I_{i, 1} \cup I_{i, 2}\right)\left(t_{i}, \delta_{0}\right)} q(s) d s
$$

where $I_{i, j}\left(t_{i}, \delta_{0}\right)=I_{j}(t, \delta) \cap\left[t_{i}, t_{i+1}\right]$ for $i=0, \ldots, k-1$ and $j=1,2$. Noting that $t_{i} \rightarrow \infty$ as $t \rightarrow \infty$, we have from (3.2) that

$$
\lim _{t \rightarrow \infty} \int_{\left(I_{i, 1} \cup I_{i, 2}\right)\left(t_{i}, \delta_{0}\right)} q(s) d s=0 \quad \text { for } i=0, \ldots, k-1 .
$$

Combining (3.4) and (3.5) we obtain (3.3). In consequence of (3.3) we see that

$$
\lim _{t \rightarrow \infty}\left(\delta \int_{\left(I_{1} \cup I_{2}\right)(t, \delta)} q(s) d s\right)=0
$$

for all $\delta>0$ and any disjoint subintervals of $[t, t+\delta], I_{1}$ and $I_{2}$. The result follows from Theorem 3.1. 
Lemma 3.1 [6]. If equation (1.1) is the limit circle, then it is oscillatory and the distance between consecutive zeros of any solution approaches zero as $t \rightarrow \infty$.

Corollary 3.1. If the conditions of Theorem 3.1 or Theorem 3.2 hold, then equation (1.1) is limit point.

The proof of this result is immediate from Lemma 3.1.

Example 3.1. Consider the equation

$$
y^{\prime \prime}+(\cos \phi(t)) y=0
$$

where $\phi \in \mathbb{C}^{1}: \mathbb{R}^{+} \rightarrow \mathbb{R}, \phi^{\prime}(t)$ is increasing, and $\phi^{\prime}(t) \rightarrow \infty$ as $t \rightarrow \infty$.

Conclusion 3. The distance between zeros of any solution of (3.6) tends to infinity as $t \rightarrow \infty$, as long as the equation is oscillatory. Hence the equation (3.6) is the limit point.

Proof. Let $\left\{t_{n}\right\}_{n=1}^{\infty}$ be the sequence of zeros of $q(t)=\cos (\phi(t))$ such that $\phi\left(t_{n}\right)=n \pi+\frac{\pi}{2}$. By the Mean Value Theorem

$$
\pi=\phi\left(t_{n+1}\right)-\phi\left(t_{n}\right)=\phi^{\prime}\left(\zeta_{n}\right)\left(t_{n+1}-t_{n}\right)
$$

for $\zeta_{n} \in\left(t_{n}, t_{n+1}\right)$. We thus have that $t_{n+1}-t_{n} \rightarrow 0$ as $n \rightarrow \infty$. For a fixed $\delta_{0}>0$ and for any $t \geq t_{2}$, let $k$ be the least integer such that $t_{k} \geq t$. Then for two given disjoint subintervals $I_{1}$ and $I_{2}$ of $\left[t, t+\delta_{0}\right]$, we have

$$
\begin{aligned}
\left|\int_{\left(I_{1} \cup I_{2}\right)\left(t, \delta_{0}\right)} q(s) d s\right| & =\left|\int_{\left(I_{1} \cup I_{2}\right)\left(t, \delta_{0}\right)} \cos \phi(s) d s\right| \\
& \leq 2\left|\int_{t_{k-1}}^{t_{k}} \cos (\phi(s)) d s\right| \leq 2\left(t_{k}-t_{k-1}\right)
\end{aligned}
$$

and $\lim _{t \rightarrow \infty} \int_{\left(I_{1} \cup I_{2}\right)\left(t, \delta_{0}\right)} q(s) d s=0$. To see this define $\theta:=\phi(s)$ and, since $\phi$ is increasing, $s=\phi^{-1}(\theta), d s=\left[\phi^{-1}(\theta)\right]^{\prime} d \theta$. We thus have

$$
\begin{aligned}
\mid \int_{t_{k}}^{t_{k}+1} \cos (\phi(s) d s \mid & =\left|\int_{k \pi+\pi / 2}^{(k+1) \pi+\pi / 2} \cos (\theta)\left[\phi^{-1}(\theta)\right]^{\prime} d \theta\right| \\
& =\left[\phi^{-1}\left(\xi_{k}\right)\right]^{\prime}\left|\int_{k \pi+\pi / 2}^{(k+1) \pi+\pi / 2} \cos (\theta) d \theta\right| \\
& =2\left[\phi^{-1}\left(\xi_{k}\right)\right]^{\prime}
\end{aligned}
$$

by the Mean Value Theorem where $\xi_{k} \in\left(t_{k}, t_{k+1}\right)$. Since $\phi^{\prime}$ is increasing, we have that $\left[\phi^{-1}(\theta)\right]^{\prime}$ is decreasing; whence $\left[\phi^{-1}\left(\xi_{k_{1}}\right)\right]^{\prime} \leq\left[\phi^{-1}\left(\xi_{k_{2}}\right)\right]^{\prime}$ whenever $k_{1} \geq k_{2}$, which implies that $\int_{t_{k}-1}^{t_{k}} \cos (\phi(s)) d s$ is a decreasing function of $k$. The conclusion follows from Theorem 3.2 and Corollary 3.1.

\section{REFERENCES}

1. W. A. Coppe, Disconjugacy, Springer, Berlin, Heidelberg, and New York, 1970.

2. B. J. Harris, On an inequality of Lyapunov for disfocality, J. Math. Anal. Appl. 140 (1990), 495-500. 
3. P. Hartman. Ordinary differential equations, 2nd ed., Birkhäuser, Boston, Basel, and Stuttgart, 1982.

4. M. K. Kwong, On Lyapunov's inequality for disfocality, J. Math. Anal. Appl. 83 (1981), 486494.

5. , On certain Riccati integral equations and second order linear oscillation, J. Math. Anal. Appl. 85 (1982), 315-330.

6. W. T. Patula and J. S. W. Wong, An $L^{p}$ analogue of the Weyl alternative Math. Ann. 197 (1972), 9-28.

7. Jurang Yan, On the distance between zeros and the limit point problem, Proc. Amer. Math. Soc. 107 (1989), 971-975.

Department of Mathematical Sciences, Northern Illinois University, DeKalb, Ilinois 60115

E-mail address: harris@math.niu.edu

E-mail address: kong@math.niu.edu 